\title{
Perioperative worries associated with low acceptance of day surgery among outpatients in Zhejiang Province, China: a survey research
}

\section{Tonghui Feng}

Zhejiang Hospital https://orcid.org/0000-0001-6150-3232

\section{Yujia Li}

Zhejiang Hospital

\section{Daipeng Zhou}

Pinghu First People's Hospital

\section{Peng Xu}

Zhejiang Hospital

\section{Xing Lu}

Zhejiang Hospital

\section{Yanfei Xia}

Zhejiang Hospital

Hongmei Wang ( $\nabla$ whm87783648@126.com )

https://orcid.org/0000-0001-9703-0940

\section{Research article}

Keywords: day surgery, outpatient surgery, ambulatory surgery, perioperative, worry, acceptance

Posted Date: September 4th, 2019

DOl: https://doi.org/10.21203/rs.2.13969/v1

License: (c) (1) This work is licensed under a Creative Commons Attribution 4.0 International License. Read Full License 


\section{Abstract}

Background Day surgery practice started rather late in mainland China, as it is not until 2001 when day surgery was officially launched. At present, Chinese patients' cognition and attitudes towards day surgery are blurred with only few relevent research. The goal of this study was to investigate the situation of outpatients' perioperative worries and its relationship with outpatients' acceptance of day surgery in Zhejiang Province, China. Methods A total of 412 outpatients in Zhejiang Hospital were evaluated by a cross-sectional questionnaire survey between January 2019 to February 2019. To evaluate outpatients' perioperative worries and acceptance of day surgery, 412 valid respondents out of all 420 outpatients were divided by 1 . demographic characteristics, 2 . been with or without worries. The correlation between worries and patient acceptance of day surgery was analyzed. Multinomial Logistic regression model was used to assess the relationship between them. Results Of the 412 outpatients, $83.5 \%$ reported perioperative worries about day surgery. Outpatients with worries were significantly older, with a higher incidence of comorbidities than those without worries. Age, educated degree, comorbidities, financial status, and surgery type were important factors of patient acceptance of day surgery. Outpatients without worries significantly scored higher in acceptance of day surgery than those with worries. The score of acceptance of day surgery was negatively correlated with perioperative worries. Compared with outpatients been worried about day surgery, those without worries had a lower risk of refusing day surgery. Conclusions The prevalence rate of perioperative worries about day surgery among Zhejiang outpatients was relatively high, and outpatients' acceptance of day surgery was negatively associated with perioperative worries. We suggest developing and utilizing a perioperative care process to address outpatients' perioperative worries, so as to improve outpatients' acceptance of day surgery.

\section{Background}

Day surgery is one of the most popular medical practices throughout the world, which is defined as an operation where the patient does not need an overnight stay by the International Association for Ambulatory Surgery (IAAS) in 2003 [1]. In Europe and North America, outpatients are usually discharged on the same working day of day surgery. However, since day surgery is in its infancy in mainland China, our current understanding of day surgery allows patients to stay over one night, while the whole process does not exceed 24 hours [2].

British surgeon Nicoll first reported on children's day surgery in 1909 [3], and the high-efficient and lowcost day surgery was propelled by the demand for reducing healthcare costs, advances in surgical technology and patients' better cognition of day surgery in Europe and North America since the 1980s. A survey in the United States showed that there were already $97 \%$ of patients being willing to choose day surgery in 1992 [4]. Nowadays, day surgery has become mainstream in most developed countries. It is reported in 2006 that day surgery account for $89 \%, 87 \%$ and $83 \%$ of all surgeries accordingly in Denmark, Spain and the United States $[5,6]$. The rates of mature types of day surgery in British hospitals are higher than $90 \%$ in 2016 [7]. However, day surgery practice started rather late in mainland China, as it is not until 2001 when day surgery was officially launched in large hospitals in Beijing, Shanghai and Sichuan 
Province. China established the China Ambulatory Surgery Alliance in 2012 which became a member of the IAAS in 2013. Furthermore, a series of policies have been issued to promote day surgery since 2015 [8]. Nevertheless, the overall proportion of day surgery in China is low at present. National Health and Family Planning Commission of China reported in 2016 that day surgery accounted for only $11 \%$ of all elective surgeries nationwide [8]. As one of the earliest pioneers in advocating day surgery in mainland China, West China Hospital of Sichuan University carried out $23.97 \%$ of all elective surgeries as day surgery [9].

Considering that the Chinese healthcare system and culture are essentially different from most developed countries, Chinese patients own their characteristics. For instance, a significant advantage of day surgery, shortening the hospital stay, may counter the tradition of many residents in China who are used to staying in hospitals till full recovery. At present, there is only few research concerning Chinese patients' cognition and attitudes towards day surgery, with local study object, limited research content, and variance in results [10-12]. Thus, it is crucial to fully understand our patients' worries and acceptance of day surgery under the circumstance of the intense doctor-patient relationship in China today, who have not yet formed a full understanding of day surgery [13]. This study was designed to identify the perioperative worries and patient acceptance of day surgery and to explore the possible association between them in Zhejiang Province, China.

\section{Methods}

\section{Patients and sampling}

Estimation of sample size was based on the assumption of a $50 \%$ incidence of worries in our patient group [11]. With $\alpha=0.05, \beta=0.05$, and $\delta=0.20$, a minimum of 210 patients was required. A total of 420 outpatients in Zhejiang Hospital were actually recruited from January 2019 to February 2019, with 412 valid respondents. The inclusion criteria were outpatients over 18 years old, being able to understand and complete the questionnaires independently, with no previous history of day surgery experience. Patients who were unable to complete the questionnaires, with poor compliance, mental disorders, or use of psychotropic drugs were excluded from the study.

\section{Study design and measurement}

The survey was conducted by a designated investigator after training. Questionnaires were given out to 420 outpatients before they had any day surgery and were collected on-site after independent completion. The investigator introduced to outpatients of the day surgery concept and basic process, explained what needs to be filled out in the questionnaires. Outpatients having difficulty in reading or writing got assistance from the investigator without interference of comprehension. If an outpatient did not understand the investigated content, the investigator would explain to them and the interpretation would be consistent. 
Out of all 420 outpatients, 412 completed all three parts of the questionnaire, including: (1) background information covering the outpatient's age, sex, educated degree, comorbidities (including hypertension, diabetes mellitus, coronary heart disease, hepatic or renal dysfunction and tumors), surgery type and financial status; (2) status of perioperative worries of day surgery (outpatients choose "Yes" if perioperative processes of day surgery worry them, otherwise, "No"), stages of day surgery process that outpatients' worry exists, and specific reasons causing such worry; (3) we designed an evaluation tool imitating Numeric Rating Scale for pain, rated patient acceptance of day surgery on an 11-point scale ranging from 0 (complete refusal) to 10 (full acceptance). Patient acceptance improves as the number increases (0-3 represent rejection, 4-6 represent a wavering attitude, 7-10 represent a positive attitude).

\section{Statistical analyses}

Two researchers entered data independently and created an original database using Microsoft Excel. Data analyses were performed using IBM SPSS Statistics for Windows (version 19.0; IBM Corp, New York, NY, USA). All statistical tests were two-sided and $p<0.05$ was regarded as statistically significant. Categorical variables were presented as frequencies and percentages and continuous variables were presented as means and standard deviations. (1) A Chi-square test was applied to compare the different status of perioperative worries according to outpatients' demographic characteristics. (2) Mann-Whitney U test was used to compare the acceptance scores between two groups divided by outpatients' demographic characteristics, Kruskal-Wallis test was used between three or more groups. (3) The correlation between perioperative worries and patient acceptance was analyzed using Spearman analysis, and a multinomial logistic regression model was used to assess the relationship between them, with the criteria for entry and deletion being 0.05 and 0.10 , the test level a being 0.05 .

\section{Results}

Four hundred and twenty questionnaires were distributed and $98.1 \%$ (412) returned as valid. The demographic characteristics, perioperative worries and patient acceptance of 412 outpatients are summarised in Table 1.

\section{Demographics of studied patients}

The age of sample patients ranges from 18 to 94 years, with a mean age of $48.27 \pm 19.22$ years. Outpatients with lower education levels, including junior high school and high school diploma, account for $70 \%$ of all 412 outpatients. Totally, 198 (48.1\%) outpatients have one or more comorbidities. Only 28 $(6.8 \%)$ outpatients considered themselves to be in poor economic condition.

\section{Situation of outpatients' perioperative worries}

Table 1 shows the number of outpatients having perioperative worries about day surgery for different demographics and medical categories. In all, 344 study participants chose "Yes" in having worries, with 
the percentage of $84 \%$ (344/412). Table 1 also shows that age, education, comorbidity, and economic status significantly influence outpatients' status of perioperative worries.

The person-time of patients having worries during preoperative and postoperative periods presented to be similar, being 334 and 359 . Specific reasons during preoperative periods include: preoperative examinations not being done timely (345), longer commuting time (156), lack of necessary preoperative instruction and education (230). In the postoperative stage, patients' worries included: unable to monitor and manage complications and adverse events (330), hard to communicate with doctors in a timely fashion (244), lack of adequate care (219), unguided disease prevention (173), inconvenient transportation and accommodation (141), healthcare payment (67), uncertain medical capacity of community hospitals (99).

\section{Patient acceptance of day surgery}

The median score of 412 outpatients' acceptance of day surgery was 6 . Outpatients' acceptance of day surgery was significantly correlated with age, educated degree, comorbidities, economic status and surgery type (Table 1). Outpatients without worries significantly scored higher in acceptance of day surgery than those with worries (Table 2).

\section{Relationship between outpatients' perioperative worries and acceptance of day surgery}

The score of acceptance of day surgery was negatively correlated with perioperative worries (Table 3). In the multivariate analysis performed by the multinomial Logistic regression model, after adjusting age, comorbidities, economic status, and surgery type, outpatients without worries had a lower risk of refusing day surgery compared with those with worries (Table 4).

\section{Discussion}

Our study shows that outpatients in Zhejiang Hospital have relatively high levels of worries about day surgery, similar to previous studies in mainland China. $84.5 \%$ of respondents were worried about perioperative periods of day surgery, which was perceived as an innovative medical service in mainland China. Yu Deliang proposed that patients and families generally required a high level of professional nursing care during the postoperative period. However, such a high level of care may be absent in the outpatient setting, therefore, patients and their families undergoing day surgery may worry.[14] According to a survey in a Shanghai community, residents' perceptions of day surgery were poor, more than $70 \%$ of community residents had doubts about day surgery.[10] An investigation in West China Hospital showed that more than $60 \%$ of patients undergoing day surgery did not understand the concept of day surgery, including how it works and its values.[11] However, those studies did not explore which population is at risk of being worried. In our study, we identified several risk factors. Patients who were older and had more systemic diseases tend to worry more in the day surgery setting. This is consistent with a Swedish study showing that older patients had more concerns about anesthesia and surgery during day surgery. Also, lower levels of education and worse economic status lead to more worries. Therefore, we suggest 
hospitals establish a standardized process to screen out patients who may have more worries, and develop strategies for this targeted patient population.

Moreover, we comprehensively investigated the specific reasons for patients' concerns. Before receiving any day surgery, outpatients worried about both preoperative and postoperative periods, and the worries covered almost all possible aspects of preoperative preparation, transportation and accommodation, rehabilitation, healthcare payment, etc. That is, outpatients believe when they are not in the hospital, it is difficult to obtain professional guidance and relevant information in time, which suggests that an effective perioperative communication mechanism between doctors and patients is absent. Studies in France and America focusing on perioperative communication channels suggested that better doctorpatient communication is correlated with higher patient satisfaction and better outcomes.[15-17] Similarly, studies in mainland China also indicated that establishing a good communication channel could promote doctor-patient communication, thus reducing patients' perioperative worries.[18] Nanjing Drum Tower Hospital applied extended nursing management during the perioperative period for patients undergoing laparoscopic day-surgery, based on the WeChat platform- one of the most popular instant messengers in mainland China, relieved the anxiety of patients effectively.[19] For those reasons, it is significant to establish a convenient, immediate and effective doctor-patient communication mechanism when patients are outside the hospital.

Patient acceptance of day surgery in our study is different from both domestic and foreign studies, the proportion of outpatients willing to undergo day surgery (scores $7-10$ ) was $35.2 \%$. While a study by Dai Yan in Sichuan Province showed that $94.5 \%$ of day-surgery patients are open to day surgery in 2016,[11] yet a recent survey in 2019 revealed that $24.88 \%$ of residents in a Shanghai community accept day surgery.[10] These diversified results may derive from different samples in those studies and unbalanced development of day surgery across China. Furthermore, compared to western countries where day surgery is highly popular, Zhejiang outpatients' acceptance of day surgery is fairly low. Given that people's attitude towards a medical practice has much to do with the development time of the medical service, healthcare insurance system, medical technology, and national health literacy,[20] patients' different attitudes may originate from the differences in above all aspects between China and developed countries.

The multivariate analysis revealed that patient acceptance of day surgery in this study was related to a variety of personal factors, suggesting that patients with younger age, higher education, no underlying disease, and better financial ability are more likely to accept the concept of day surgery. These findings raised a possibility that with the improvement of national education level, physical quality and economic ability, Chinese patients will gradually be more open-minded about day surgery. Besides, surgery type also affects patient acceptance of day surgery. For example, orthopedic outpatients are relatively more reluctant to take operations as day surgery, we consider this may result from Chinese patients' traditional thinking that patients should have a full recovery in the hospital. Orthopedic surgeries are perceived as extensive and complex, patients undergoing orthopedic surgeries think they should recover in the hospital, not in the day surgery setting. Under such circumstances, hospitals should improve patient education according to Chinese patients' characteristics to promote day surgery. 
Although there are studies focusing on patients' worries and patient acceptance of day surgery, the possible association between them is blurred. Our study implies that patient acceptance of day surgery is influenced by their perioperative worries. In other words, patients who had fewer worries had a higher level of acceptance of day surgery. Accordingly, sufficient doctor-patient communication and interaction need to be guaranteed by strengthening perioperative systems of day surgery, focusing on building communication channels to provide patients timely, effective and professional guidance, so that to dispel worries of patients during the perioperative period, thereby increase patient acceptance of day surgery.

\section{Limitations}

The major drawback of the study lies in the sample taken from one center. Nevertheless, whether other patients like inpatients and patients who have experienced day surgery are of the same situation needs further investigation. Moreover, more researches are warranted not only in Zhejiang province but also across China to provide more evidence for present patient attitude towards day surgery in mainland China. Second, we used a subjective method to assess the patient acceptance of day surgery, similar to previous studies that also utilized various subjective evaluations. However, whether those subjective methods are reliable and valid need further proof. Third, future studies could investigate how to establish an effective doctor-patient communication mechanism to address patient worries during perioperative periods so that to increase patient acceptance of day surgery.

\section{Conclusion}

Our study shows that the majority of outpatients in our hospital are worried about day surgery, for both pre and postoperative issues, ranging from preoperative preparation, transportation to postoperative accommodation, rehabilitation, healthcare payment, etc. Zhejiang outpatients' acceptance of day surgery is relatively low and the low acceptance is highly correlated with various worries the patients have.

Establishing an effective perioperative doctor-patient communication channel may help alleviate patients' worries and promote day surgery in Zhejiang Province.

\section{Abbreviations}

IAAS: International Association for Ambulatory Surgery

\section{Declarations}

\section{Acknowledgements}

Not applicable.

\section{Funding}


This study is supported by the Key Project of the Chinese Ministry of Health and Medical Scientific Research Foundation of Zhejiang Province (No.WKJ-2J-1708) in the design of our study and collection, analysis, and interpretation of data and in writing the manuscript.

\section{Availability of data and materials}

The datasets used and analyzed in our study are available from the corresponding author on reasonable request.

\section{Authors' contributions}

HW and TF conceived and designed the study, conducted the survey, analyzed and interpreted the data, and wrote the manuscript.

All of the authors participated in the study design and revised the manuscript, they have read and approved the manuscript.

\section{Ethics approval and consent to participate}

The study was approved by the Ethics Committee of Zhejiang Hospital with decision number: 24K. Before the survey, a written informed consent form was obtained from all respondents to participate in the study.

\section{Consent for publication}

Consent for publication was obtained from all the study participants.

\section{Competing interests}

The authors declare that they have no competing interests.

\section{References}

1. International Association for Ambulatory. Surgery. http://www.iaas-med.com/index.php/iaasrecommendations/extended-recovery-facilities

2. China Ambulatory Surgery Alliance. http://www.chinaasa.org/Content/index/id/1777

3. M NJ: The surgery of infancy. BMJ 1909, 11:753-756.

4. Philip BK: Patients' assessment of ambulatory anesthesia and surgery. Journal of Clinical Anesthesia 1992, 4(5):355-358.

5. P KM: The Nordic Health Care Model. In: The Nordic Health Care Model 9th International Congress on Ambulatory Surgery. Copenhagen; 2011.

6. Philip BK: Day Care Surgery:The United States Model of Health Care. Ambulatory Surgery 2012, 17(4):81-82. 
7. Mark Skues IJ: Ten Year Performance of Ambulatory Surgery in England. Ambulatory Surgery 2018, 24(1):15-19.

8. National Health Commission of the People's Republic of China. http://www.nhc.gov.cn

9. Y Liu HM, H Ye: Problems and countermeasures in the promotion of laparoscopic cholecystectomy in day surgery [in Chinese]. Chinese Journal of Hospital Administration 2017, 33(5):354-356.

10. YT Wu LY: Investigation and Analysis of Cognition of Day Surgery by Residents in a Shanghai Community [in Chinese]. Chinese General Practice Nursing 2019, 17(2):149-153.

11. Y Dai YZ, HS Ma, ZC Li: Investigation and analysis of patient acceptance and satisfaction of day surgery [in Chinese]. West China Medical Journal 2016, 31(4):639-641.

12. Yu WP CY, Duan GM, Hu H, Ma HS, Dai Y: Patients' perceptions of day surgery: a survey study in China surgery. Hong Kong Med J 2014, 20(2):134-138.

13. X Bai HM, L Luo: Research and Prospect of Comparison Between China and Abroad in the Day Surgery Development [in Chinese]. Chinese Hospital Management 2014, 34(5):35-37.

14. DL Yu XL, PT Ning, QC Zhao: Issues Need to Discussion about Ambulatory Surgery in the Current China [in Chinese]. Medicine and Philosophy 2015, 36(3B):1-3.

15. Armstrong KA CP, Brown M, Beber B, Semple JL: Effect of Home Monitoring via Mobile App on the Number of In-Person Visits Following Ambulatory Surgery: A Randomized Clinical Trial. JAMA Surg 2017, 152(7):622-627.

16. Carrier G CE, Beyer-Berjot L, Faucheron JL, Joris J, Slim K: Post-discharge follow-up using text messaging within an enhanced recovery program after colorectal surgery. J Visc Surg 2016, 153(4):249-252.

17. van Eck CF TA, Banffy MB, Gambardella RA: Web-Based Education Prior to Outpatient Orthopaedic Surgery Enhances Early Patient Satisfaction Scores: A Prospective Randomized Controlled Study. Orthop J Sports Med 2018, 6(1):2325967117751418.

18. LL Zhan QJ, W Ji, ZL Song, CX Qin The Application of Medical Network Platform in the Perioperative Nursing of Day Surgery of Sleeve-type Foreskin Circumcision [in Chinese]. Chinese Journal of Aesthetic Medicine 2018, 27(3):134-136.

19. YL Li NL, ZT Fang, Y DIng, XL Huang: The extended nursing management based on WeChat platform during perioperative period for patients undergoing laparoscopic day-surgery [in Chinese]. Nursing of Integrated Traditional Chinese and Western Medicine 2018, 4(2):37-40.

20. Hälleberg Nyman M NU, Dahlberg K, Jaensson M: Association Between Functional Health Literacy and Postoperative Recovery, Health Care Contacts, and Health-Related Quality of Life Among Patients Undergoing Day Surgery: Secondary Analysis of a Randomized Clinical Trial. JAMA Surg 2018, 153(8):738-745.

\section{Tables}

TABLE 1. Patient demographic data, perioperative worries and patient acceptance $(n=412)$ 


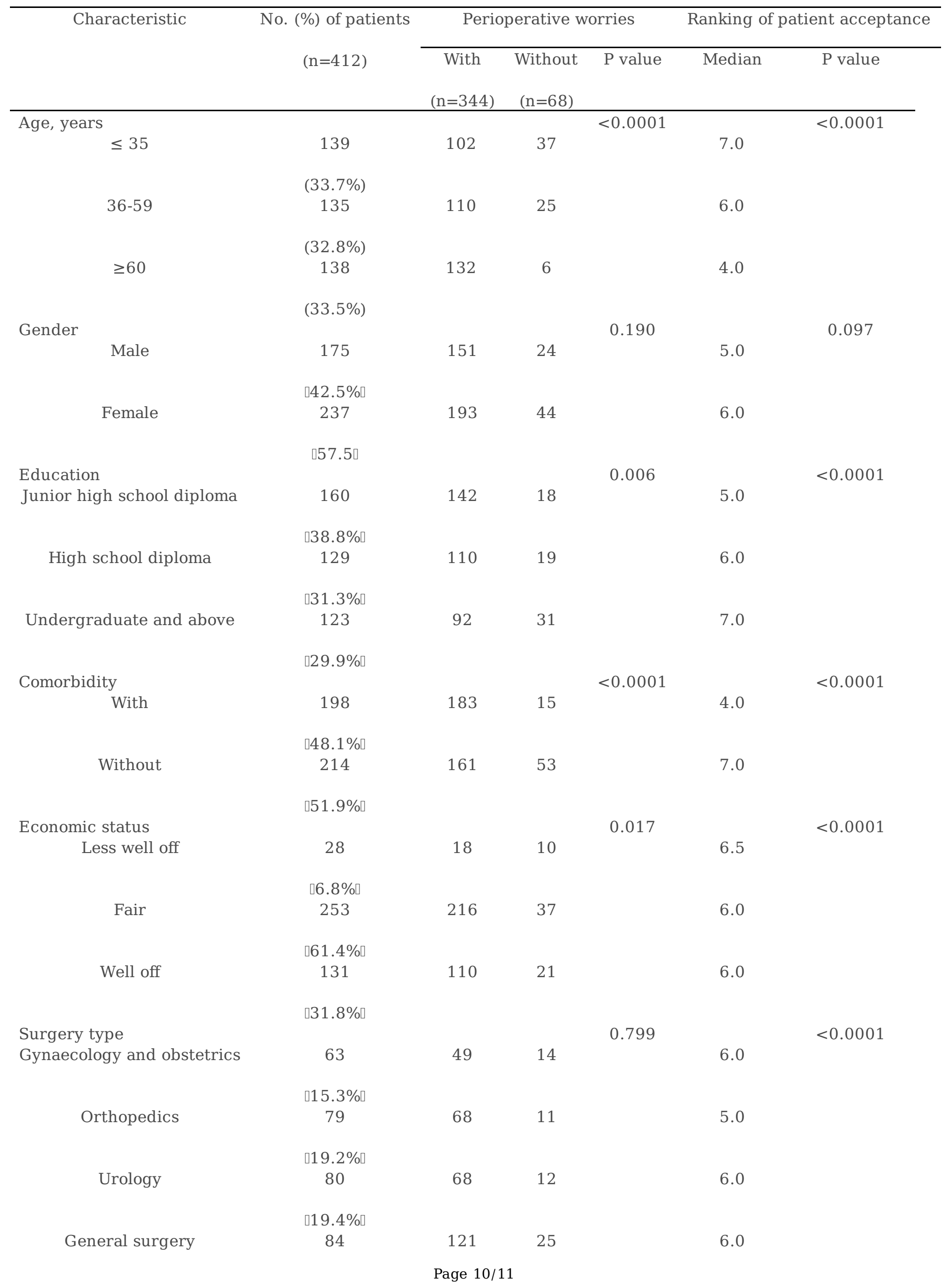


Gastroenterology

Ear, nose, throat and eye
$020.4 \% \square$

62

$\square 15.0 \% \square$

44
51

38
11

5.0

$\square 10.7 \% \square$

\section{TABLE 2. Score of patient acceptance of day surgery between outpatients with and without}

worries (median)

\begin{tabular}{ccccc}
\hline variable & With worries $(\mathrm{n}=344 \square$ & Without worries $\square \mathrm{n}=68 \square$ & $\chi^{2}$ value & $\mathrm{P}$ value \\
\hline Score of patient acceptance of day surgery & 5.0 & 7.0 & 71.334 & $<0.0001$ \\
\hline
\end{tabular}

TABLE 3. Spearman correlation analysis of patient perioperative worries and acceptance of day surgery $(n=412 \square$

\begin{tabular}{ccc}
\hline variable & acceptance of day surgery score & P value \\
\hline Patient perioperative worries & $-0.371(\mathrm{r}$ value $)$ & $<0.0001$ \\
\hline
\end{tabular}

TABLE 4. Multinomial Logistic regression model analysis between patient perioperative worries and acceptance of day surgery $(n=412)$

\begin{tabular}{cccccccc}
\hline variable & & $\mathrm{n}$ & $\beta$ value & sx $\square$ value & Wald $\chi^{2}$ value & P value & OR (95\%CI) value \\
\hline Perioperative worries & $\begin{array}{c}\text { with } \\
\text { without }\end{array}$ & 644 & -1.568 & 0.432 & 13.162 & $<0.0001$ & 0.208
\end{tabular}

[0.089-0.486ロ

The perioperative worry was an independent variable in the multinomial Logistic regression model. Confounding factors entering the model were age, comorbidities, financial status and surgery type. 